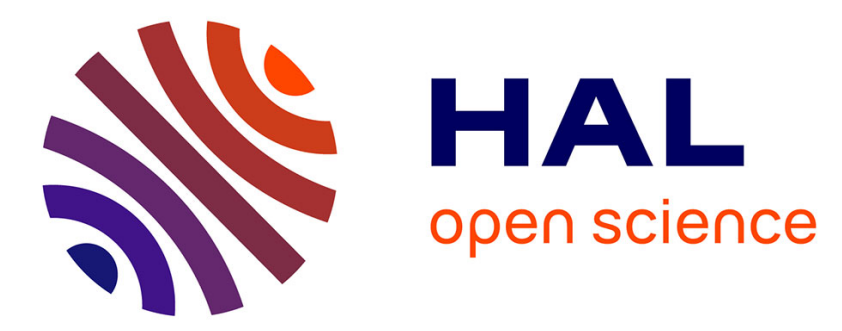

\title{
Empirical Analysis and Modeling of Bluetooth Low-Energy (BLE) Advertisement Channels
}

Ali Nikoukar, Mansour Abboud, Borna Samadi, Mesut Güne,sgüne,s, Behnam Dezfouli

\section{To cite this version:}

Ali Nikoukar, Mansour Abboud, Borna Samadi, Mesut Günessgüne,s, Behnam Dezfouli. Empirical Analysis and Modeling of Bluetooth Low-Energy (BLE) Advertisement Channels. 17th Annual Mediterranean Ad Hoc Networking Workshop (Med-Hoc-Net 2018), Jun 2018, Capri Island, Italy. pp.45-51. hal-01832527

\section{HAL Id: hal-01832527 \\ https://hal.inria.fr/hal-01832527}

Submitted on 8 Jul 2018

HAL is a multi-disciplinary open access archive for the deposit and dissemination of scientific research documents, whether they are published or not. The documents may come from teaching and research institutions in France or abroad, or from public or private research centers.
L'archive ouverte pluridisciplinaire HAL, est destinée au dépôt et à la diffusion de documents scientifiques de niveau recherche, publiés ou non, émanant des établissements d'enseignement et de recherche français ou étrangers, des laboratoires publics ou privés.

\section{(c)(1)}

Distributed under a Creative Commons Attribution| 4.0 International License 


\title{
Empirical Analysis and Modeling of Bluetooth Low-Energy (BLE) Advertisement Channels
}

\author{
Ali Nikoukar*, Mansour Abboud*, Borna Samadi ${ }^{\dagger}$, Mesut Güneş*, Behnam Dezfouli ${ }^{\ddagger}$ \\ ${ }^{*}$ Communication and Networked Systems (ComSys), Faculty of Computer Science, \\ Otto-von-Guericke University, Magdeburg, Germany \\ ${ }^{\dagger}$ College of Engineering, University of Tehran, Iran \\ $\ddagger$ Internet of Things Research Lab, Department of Computer Engineering, Santa Clara University, USA \\ \{ali.nikoukar, mansour.abboud, mesut.guenes\}@ovgu.de, bornasamadi@ut.ac.ir, bdezfouli@scu.edu
}

\begin{abstract}
Bluetooth Low Energy (BLE) is a widely-used lowpower wireless standard in the Internet of Things (IoT) domain. This standard provides a set of advertisement channels, which are primarily used for device discovery, connection initiation, and information broadcast. Beacon transmission over these advertisement channels is the enabler of applications such as indoor positioning, product advertisement, and medical monitoring. Meanwhile, the performance and accuracy of these applications highly depend on the characteristics of communication over advertisement channels. Unfortunately, the existing literature does not offer an extensive characterization of these channels under various operational conditions. In this paper, we address this research gap through conducting extensive experiments in four different environments. We study the effect of environment and interference on noise floor and signal propagation, and we present a model for noise floor and extract the parameters of lognormal path loss model. The proposed models, in particular, can be directly used in simulation tools for modeling BLE wireless channels as well as applications such as indoor positioning.

Index Terms-Bluetooth Low Energy (BLE), Log-Normal Shadowing Model (LNSM), multi-path fading, Bluetooth beacon, channel modeling, simulation.
\end{abstract}

\section{INTRODUCTION}

As the Internet of Things (IoT) strives to connect every object to the Internet [1], a variety of wireless technologies are involved to obtain this goal. To fulfill the requirements of IoT, an applicable technology must be low-power, available, inexpensive, reliable, and provide mechanisms to support coexistence. To this end, Bluetooth Low Energy (BLE) is a widely-adopted standard that satisfies the aforementioned requirements.

Introducing iBeacon by Apple in 2013 [2] expanded the application domain of BLE through adding advertisement and localization capability. Nowadays, Indoor Positioning System (IPS) is one of the major applications of BLE. It is reported that $75 \%$ of top US retailers have already deployed beacons in their facilities, and $84 \%$ of international airports will be using BLE beacons by 2019 [3]. Commercial products like StickNFind [4], TrackR [5] and Estimote [6] exploit BLE beacons. These products offer various types of services such as patient monitoring, navigation in shopping malls, broadcasting information in train stations or museums, and finding lost items. In 2016, the global market for indoor positioning service

ISBN 978-3-903176-05-8 @ 2018 IFIP was $\$ 5.22$ billion and it is expected to grow up to $\$ 40.99$ billion by 2022, with an annual growth rate of $42.0 \%$ [7].

Although various wireless channel models exist for Bluetooth classic [8], 802.11 [9]-[12] and 802.15.4 [13], [14] standards, the wireless channel of BLE has not been investigated thoroughly as it is a relatively new standard. In particular, the models proposed for Bluetooth classic are not applicable to BLE because of the differences in their physical layer implementation. For example, the channel bandwidth and modulation used by BLE and Bluetooth classic are different. As BLE is particularly suitable for IoT applications, its wireless channel characterization is important due to two reasons: First, applications such as IPS perform range estimation based on the signal strength received from beacon sources. Second, the accuracy of the wireless channel model used by simulation tools directly affects the performance of protocols developed.

In this paper, we focus on the characterization of BLE Advertisement Channels (ADV_CHs) and propose models for various environments. Specifically, the contribution of this paper is three-fold: First, this research is the first experimental evaluation of BLE's ADV_CHs in various environments considering a variety of influential factors, including interference, environmental size, and the existence of obstacles and reflective objects. Second, we study the effect of 802.11 interference on the stability of noise floor perceived by BLE devices on the three advertisement channels. Third, the obtained results are used to extract the parameters of Log-Normal Shadowing Model (LNSM) under various conditions. We also highlight the shortcomings of LNSM and identify areas of future research. The proposed models enable the research community to integrate accurate channel models of BLE's ADV_CHs in various applications (such as IPS) and simulation tools to achieve realistic performance evaluations.

The remainder of this paper is organized as follows. A brief overview of BLE's physical layer is presented in Section II. In Section III, the methodology of research including LNSM, noise floor definition, experimental setup, and experimental parameters, are explained. Section IV presents and analyzes the obtained results. Section V elaborates the background of the research on BLE ADV_CHs. Finally, we conclude the paper in Section VI. 


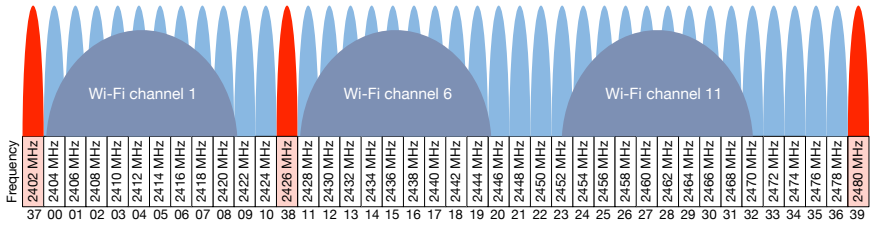

Fig. 1: The $2.4 \mathrm{GHz}$ frequency band shared by BLE and 802.11. The red channels are the advertisement channels used by BLE beacons.

\section{WIRELESS COMMUNICATION USING BLE}

High power consumption is one of the most well-known challenges of wireless technologies for IoT. Although Bluetooth was originally used for file sharing and audio streaming, in 2010, Bluetooth Special Interest Group (SIG) made a remarkable change in its protocol stack and introduced BLE. The new standard paved the way towards new IoT applications such as IPS.

Among other changes, BLE employs a new physical layer design, which results in lower energy consumption. In contrast with Bluetooth classic which uses 79 channels each $1 \mathrm{MHz}$ wide, BLE uses 40 channels each $2 \mathrm{MHz}$ wide. BLE employs Gaussian Frequency Shift Keying (GFSK) modulation, and the transmit power is within -20 to $+20 \mathrm{dBm}$. BLE classifies the channels into two groups: advertisement channels (ADV_CHs) and data channels, as Figure 1 shows. ADV_CHs are responsible to broadcast advertisement Protocol Data Units (PDUs), and data channels are only used for data exchange. Based on the broadcasted PDU over ADV_CHs, the device either establishes a connection and switches to the data channels, or continues a one-way information sharing. The unique channel assignment of ADV_CHs tries to minimize interference with 802.11. As Figure 1 shows, the placement of these channels minimizes interference with channel 1, 6, and 11 of $802.11 \mathrm{~b} / \mathrm{g} / \mathrm{n}$ standard, which are the widely-used and nonoverlapping channels in the allocated frequency spectrum of this standard [15].

RF signals sent through ADV_CHs experience path loss and multipath effect, which result in signal attenuation and variations, respectively. Path loss and multipath are caused by factors such as obstacles, noise floor, and signal reflection off the walls and objects. Both path loss and multipath affect the performance of applications such as IPS. For example, IPS relies on the received signal strength value of the signals received to estimate the distance from the sources of beacon broadcast. If the estimation is not accurate, the triangulation algorithm fails to compute an accurate location. Accordingly, it is essential to characterize and model ADV_CHs to facilitate the design and development of reliable beacon-based applications.

\section{Methodology: Modeling And Experimentation}

In this Section, we explain the path loss model used as well as the experimentation methodology employed.

\section{A. Log-Normal Shadowing Model (LNSM)}

As mentioned earlier, signal path loss is an essential characteristic of wireless channels. To this end, various models have been proposed to compute the strength of the signal received from a transmitter sending $d$ meters away. This value is usually referred to as received signal strength (RSS). The three well-known models of signal decay are [16]: (i) freespace propagation model, (ii) two-ray model, and (iii) lognormal shadowing model (LNSM). The free-space propagation model simply represents received signal power in an obstaclefree environment. In the two-ray model, in addition to the lineof-sight (LoS) signal, the ground reflected signal is included as well. Both of these models represent signal strength as a fixed function of distance. This representation results in a circular communication range, which is referred to as the unit disk graph model. However, as existing studies indicate [13], RSS around a sender shows variations that are best modeled through a normal distribution. These variations are caused by the signals reflected off the walls and objects in the environment. LNSM represents path loss and signal variations as follows:

$$
P L(d)=P L\left(d_{0}\right)+10 \eta \log _{10}\left(\frac{d}{d_{0}}\right)+N\left(0, \sigma_{c h}\right)
$$

where $P L(d)$ represents path loss at distance $d, P L\left(d_{0}\right)$ is the path loss at the reference distance $d_{0}, \eta$ is the path loss exponent that indicates the rate at which path loss increases versus distance $d$, and $N\left(0, \sigma_{c h}\right)$ is a zero-mean Gaussian random variable with standard deviation $\sigma_{c h}$. In other words, $\sigma_{c h}$ represents the standard deviation of signal power fluctuations caused by multi-path.

The parameters of LNSM depend on both environmental factors (e.g., obstacles, reflective surfaces, and humidity) and characteristics of RF transceiver (e.g., frequency band, transmit power, and modulation scheme). Consequently, empirical measurements are necessary to extract these parameters. However, as environmental factors do not stay unchanged over time, we need to estimate the variations of signal power over time. To this end, we applied the trust region reflective least squares [17] curve fitting algorithm.

\section{B. Noise Floor}

When external factors are neglected, the noise floor of a transceiver is modeled through computing its noise figure, which is a function of temperature and noise bandwidth. On the other hand, interference shows temporal variations and affects the RSS perceived from a receiver's point of view. In fact, the signal to interference-and-noise ratio (SINR) perceived by a receiver is modeled as $S I N R=S /(N+I)$, where $S$ is the signal power, $N$ is noise figure, and $I$ is interference. In order to estimate RSS accurately, we need to measure both noise figure and interference in various environments. To this end, we used a receiver to collect RSS samples when no other BLE node is transmitting. RSS samples in an interference-free environment represent noise figure, and RSS samples in an environment with 802.11 devices represent 

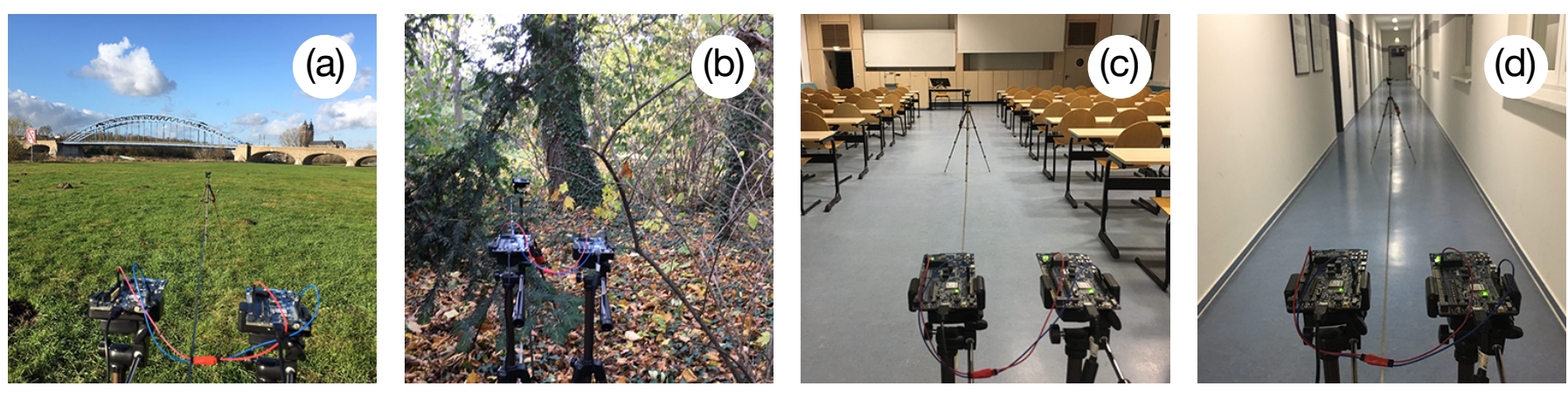

Fig. 2: Experimental environments: (a) is an open field park with line-of-sight (LoS) transmission, (b) is a forest with blocked LoS, (c) is a classroom located in a university building, and (d) is a corridor at the same university building. Environment (c) and (d) include random people movement during experimentation.

noise figure plus interference level. In this paper, we refer to the term $N+I$ as noise floor. After computing the noise floor of various environments, we estimate the actual signal power received from a sender through subtracting noise floor from the signal power received. Therefore, $R S S_{d B}=P_{r}-N-I=$ $P_{t}-P L(d)-N-I$, where $P_{t}$ and $P_{r}$ are transmission and reception power, respectively.

\section{Environments}

We have conducted extensive experiments in four different environments. Figure 2 shows these environments. As it can be observed, there are two outdoor environments and two indoor environments, as follows:

- Environment (a). A park with LoS transmission, free of obstacles and operating wireless devices.

- Environment (b). A forest with low foliage density, selected to observe the effect of trees and plants between the sender and receiver. The environment is free of interference signals.

- Environment (c). A classroom located in a university building. The benches and chairs do not block Line-ofSight (LoS) transmission. However, to represent a realworld scenario, we did not avoid people movement during the experimentation. Additionally, several 802.11 APs operate in this area.

- Environment (d). A corridor located on the third floor of the university building. There are windows on the two sides of the corridor. Furthermore, along the left side of the corridor there are three rooms in which 802.11 APs are operating. Similar to the environment (c), random student movement happened during the experimentation.

For simplicity, we refer to the environments by the labels shown in Figure 2. Table I summarizes the characteristics of these environments based on our measurements. In this table "Number of Locations" refers to the number of experimentation points used in that environment.

\section{Hardware Setup}

We used nRF52840 SoC [18] as the BLE sender and receiver. This platform supports low-power wireless technologies such as BLE 5.0, ANT, IEEE 802.15.4, and $2.4 \mathrm{GHz}$ proprietary protocols. The SoC includes an ARM Cortex-M4
Table. I: Environmental Parameters

\begin{tabular}{l|llll}
\hline \multirow{2}{*}{ Parameter } & \multicolumn{4}{|c}{ Environment } \\
\cline { 2 - 5 } & (a) & (b) & (c) & (d) \\
\hline Ambient Temperature $\left[{ }^{\circ} \mathrm{C}\right]$ & 11.0 & 14.8 & 21.0 & 21.1 \\
Relative Humidity [\%] & 58.1 & 53.4 & 48.9 & 49.0 \\
Area $\left[\mathrm{m}^{2}\right]$ & Open & Open & $18 \times 12$ & $31 \times 1.8$ \\
Number of Locations & 11 & 11 & 15 & 15 \\
Packet Size & $9 \mathrm{Byte}$ & $9 \mathrm{Byte}$ & $9 \mathrm{Byte}$ & $9 \mathrm{Byte}$ \\
Beacons/Distance & 1000 & 1000 & 1000 & 1000 \\
\hline
\end{tabular}

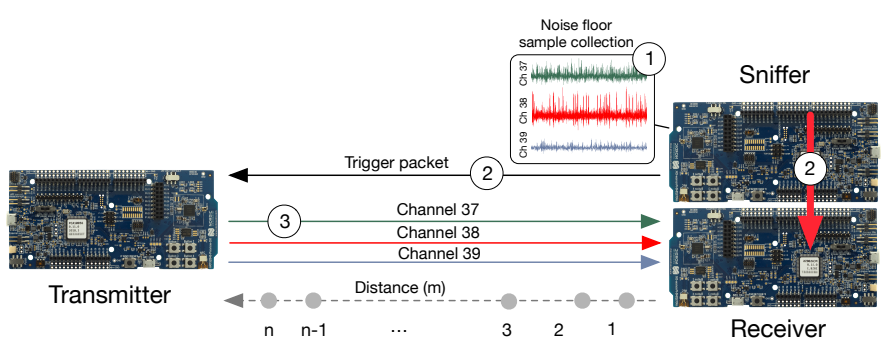

Fig. 3: Communication scenario: (1): Sniffer captures 40,000 noise floor samples in a single ADV channel. (2): Sniffer sends a trigger packet to the transmitter and configures (using a wire link) the receiver to listen to a certain ADV channel. (3) Transmitter starts sending packets on the specified ADV channel and the receiver collects the packets. This process repeats for all the ADV channels.

processor with $1 \mathrm{MB}$ flash memory and 256KB RAM. The BLE transceiver of this platform presents receiver sensitivity $-95 \mathrm{dBm}$ and supports transmit powers in range -20 to $+8 \mathrm{dBm}$. Note that sensitivity does not represent the minimum signal level detectable; instead, it indicates that if the received power of an input signal is $-95 \mathrm{dBm}$, bit error rate $10^{-3}$ is achieved during communication.

Our testbed setup includes three boards: sender, receiver, and sniffer. Each node is placed on a tripod with height 1 meter. The broadcaster and receiver exchange 1000 packets for each distance-channel configuration. Before exchanging packets between the broadcaster and receiver, the sniffer is used to measure the noise floor. To this end, before each experiment, in each environment a total of 40,000 samples were collected. The inter-sampling interval is $9 \mu \mathrm{s}$. After measuring the noise floor, the sniffer sends a trigger message to the sender to start packet broadcast (Figure 3). 


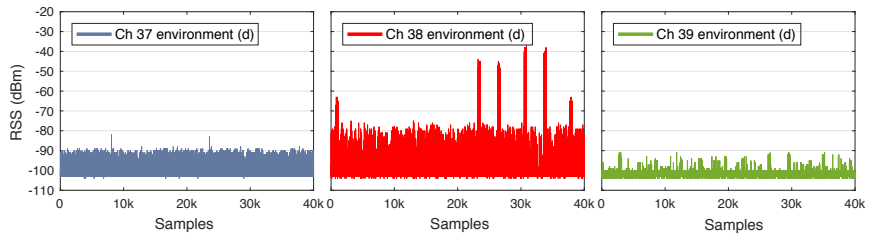

Fig. 4: Noise floor of the classroom (environment (d)) on channel 37, 38 and 39. The interference of channel 38 is the highest due to its overlap with two 802.11 channels.

\section{EMPIRICAL RESULTS}

In this Section, we present and discuss the empirical data collected for noise floor and RSS modeling.

\section{A. Noise Floor}

Although the parameters of LNSM are essential for RSS modeling, the effect of noise floor should be accounted as well because it directly contributes to a node's perception of reception power. Noise floor measurement results are summarized in Table II. In this table, $\mu$ refers to the mean noise floor measured, and $\sigma$ represents the standard deviation of noise floor variations.

As it can be observed in Table II, the average noise floor is almost similar for all the environments, however, channel 37 and 38 show significantly higher variations in environment (c) and (d), which is due to 802.11 interference. Figure 4 demonstrates noise floor samples collected on channel 37, 38 and 39 in environment (d). This figure represents channel 39 as the most reliable and clear channel, and in contrast, both channel 37 and 38 show higher noise floor. Although channel 37 and 38 do not overlap with the central frequency of 802.11 channels, their frequency is very close to 802.11's channel 1 and 6 , respectively. We clarify this issue through Figure 5. As the figure shows, for example, BLE's channel 37 overlaps with the side lobe of 802.11's channel 1. Although the power of the side mask is $20 \mathrm{dBr}$ ( $\mathrm{dB}$ relative) less than the center frequency, it may cause significant interference on BLE's channel 37 depending on the transmission power and distance from the 802.11 interferers. The same problem happens for channel 38 when 802.11's channel 6 is in use. As Figure5(a) and (b) indicate, both channel 37 and 38 show an almost similar level of overlapping with 802.11 channels. However, according to Figure 4, channel 38's noise floor represents higher variations compared with channel 37 . The reason is that, compared with channel 37 which overlaps with one 802.11 channel only, channel 38 overlaps with multiple 802.11 channels. For example, if 802.11 channel 1 and 6 are in use, then BLE's channel 38 overlaps with both, as Figure 5 (a) and (b) show. On the other hand, channel 39 overlaps with only one of the main channels of 802.11. In addition, the distance between the central frequency of BLE's channel 39 and 802.11's channel 11 is more than that of the distances for the other two BLE channels.

Noise floor variations directly impact the accuracy of applications that rely on RSS measurement, such as IPS. For
Table. II: Noise Floor Characteristics of Various Environments

\begin{tabular}{|c|c|c|c|c|c|}
\hline \multirow{2}{*}{ Parameter } & \multirow{2}{*}{ Channel } & \multicolumn{4}{|c|}{ Environment } \\
\hline & & (a) & (b) & (c) & (d) \\
\hline \multirow{3}{*}{$\mu$} & 37 & -101.1 & -100.7 & -101.0 & -101.5 \\
\hline & 38 & -101.3 & -101.1 & -101.2 & -101.5 \\
\hline & 39 & -101.7 & -101.6 & -101.3 & -101.7 \\
\hline \multirow{3}{*}{$\sigma$} & 37 & 0.74 & 0.98 & 2.26 & 1.12 \\
\hline & 38 & 0.74 & 0.88 & 2.16 & 2.54 \\
\hline & 39 & 0.77 & 0.89 & 1.96 & 1.28 \\
\hline
\end{tabular}

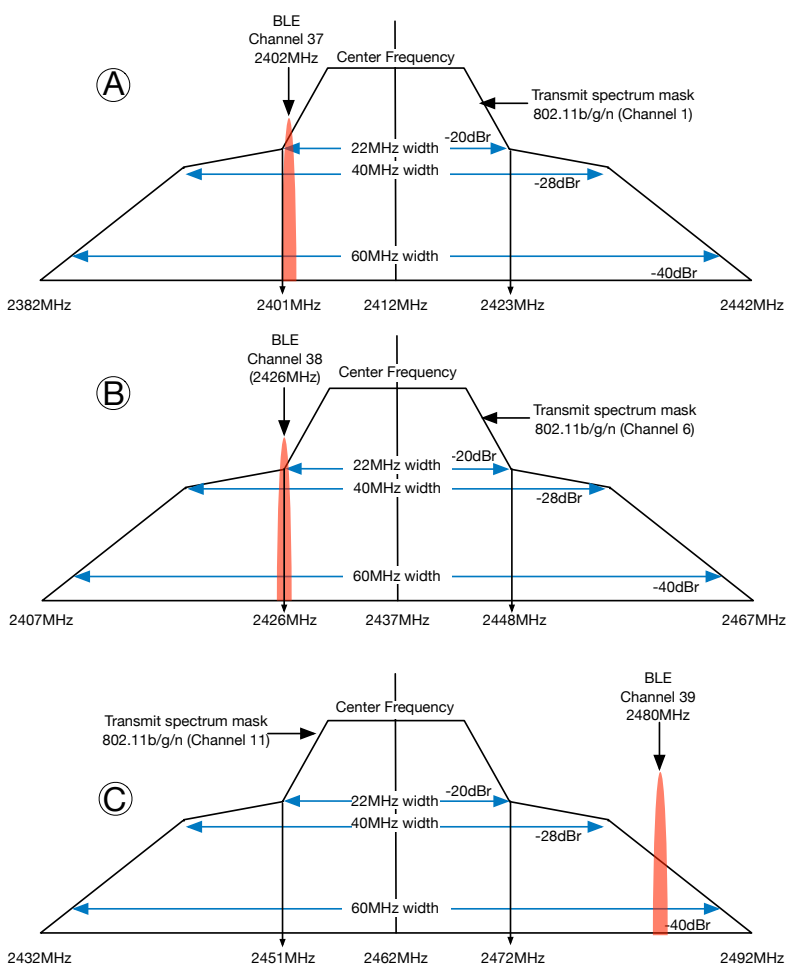

Fig. 5: The transmit mask of 802.11 channels and their overlap with BLE's ADV_CHs. BLE's channel 39 and 38 are the least and most affected channels by 802.11 interference, respectively.

example, when the variations of noise floor is high, more number of samples must be collected to estimate the actual RSS received from a sender.

\section{B. Path Loss and Shadowing}

The results of RSS measurement in outdoor and indoor environments are presented in Figure 6. The x-axis indicates the distance between sender and receiver, and the y-axis represents RSS from the receiver's point of view. Markers and error bars indicate the mean and standard deviation of RSS samples. The logarithmic lines demonstrate the best fit over the collected samples for each channel. Table III presents a summary of the parameters extracted from the experimental results. These values can be directly used by simulators or algorithms that rely on RSS-to-distance mapping.

Comparing Figure6 (a) and (b) with (c) and (d) indicates that the outdoor environments show a steady reduction in signal power versus distance, and the indoor environments show 

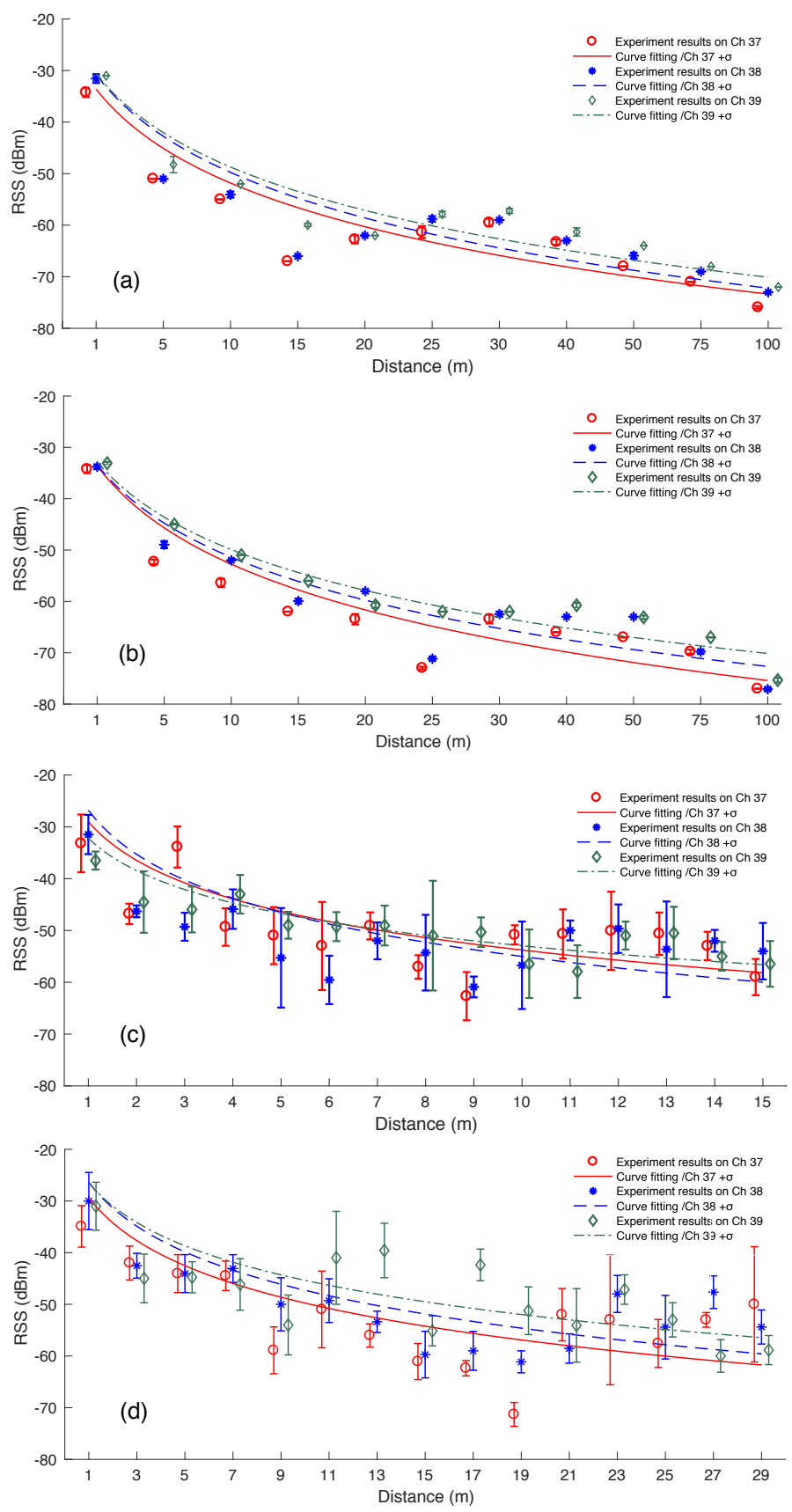

Fig. 6: Empirical measurement of RSSI versus distance. Transmission power is $0 \mathrm{dBm}$. The labels used correspond with those used in Figure 2. For example, label "(a)" in this figure represents the results collected in environment "(a)" of Figure 2.

significantly higher variations around the fitted lines. This behavior is observable per distance as well as at a larger scale when the distance from the sender increases. Although this behaviour is partly due to the shorter measurement distance of the indoor environments, there are more important reasons that we discuss as follows.

Indoor environments cause higher shadowing effect due to the existence of reflective obstacles, walls and people movement, compared to the outdoor environments. For a given distance, the stronger shadowing effect results in a significantly higher number of reflected signals received at the receiver. Overlapping a higher number of signals, in turn, may cause either signal amplification or attenuation: signal amplification occurs when the phases of the overlapping signals are similar, otherwise, attenuation happens. This behaviour can be observed in Table III. For example, in channel 39, the $\sigma$ of the open field park (i.e., 0.36 in environment (a)) is lower than that of the classroom (i.e., 4.3 in environment (c)). These results indicate that RSS-based positioning in outdoor environments is more reliable and requires a fewer number of RSS samples collected.

In addition to higher per-distance variations, at a larger scale, the indoor environments show higher variations as the distance from the sender increases. This behavior has been verified in [19] as well. Due to the existence of reflective objects, any small variation in the location of sender or receiver in the indoor environments results in a significant change of the wireless channel. For instance, a new node placement may result in a higher/lower number of signals received by the receiver, thereby, reducing/increasing path loss. Therefore, although it is expected to see lower variations of channel 39 versus distance compared to the other two channels (due to its lower 802.11 interference), the effect of node placement on wirless channel is significant and results in higher variations compared to the outdoor environments. Furthermore, in indoor environments, such as university buildings, people movement is one of the main parameters affecting path loss. In particular, we can observe the higher multipath of environment (d) compared with (c). This is because the corridor environment has a narrower width, and people movement temporarily blocks the LoS.

Another reason behind the non-steady decrease of RSS over distance is antenna anisotropy. Studies show that signal propagation around a transmitter, as well as the capability of an antenna to capture the signals arriving from different directions, are not isotropic. Hardware anisotropy has two main effects: The power of the signal sent and received depend on the direction of both sender and receiver. At some directions, the receiver antenna presents higher sensitivity and captures stronger signals. Similarly, the signal power propagated by the sender differs at various angles. For a LOS communication, these factors directly affect RSS measurement. For example, rotating the sender by $x$ degree and receiver by $y$ degree might result in the strongest LOS link possible. For indoor environments with multiple reflective objects, the effect of radio anisotropy is stronger and harder to predict because it depends on the characteristics of the wireless channel between the sender and receiver. In general, since node and people movements change the angles of signal arrivals and result in a new multipath channel, a non-logarithmic decrease in power can be observed. For example, if a higher number of multipath signals are received from a particular angle, then matching the highest sensitivity direction of the receiver's antenna with that angle results in a stronger link. Although the study of antenna anisotropy is beyond the scope of this paper, these 
Table. III: Summary of the LNSM Parameters

\begin{tabular}{|c|c|c|c|c|c|}
\hline \multirow{2}{*}{ Parameter } & \multirow{2}{*}{ Channel } & \multicolumn{4}{|c|}{ Environment } \\
\hline & & (a) & (b) & (c) & (d) \\
\hline Path Loss $(\eta)$ & $\begin{array}{l}37 \\
38 \\
39\end{array}$ & $\begin{array}{l}2.07 \\
2.15 \\
2.05\end{array}$ & $\begin{array}{l}2.17 \\
2.01 \\
1.87\end{array}$ & $\begin{array}{l}2.47 \\
2.87 \\
2.13\end{array}$ & $\begin{array}{l}2.14 \\
2.20 \\
1.98\end{array}$ \\
\hline$\sigma_{c h}$ & $\begin{array}{l}37 \\
38 \\
39\end{array}$ & $\begin{array}{l}0.39 \\
0.23 \\
0.36\end{array}$ & $\begin{array}{l}0.41 \\
0.29 \\
0.20\end{array}$ & $\begin{array}{l}4.20 \\
4.60 \\
4.30\end{array}$ & $\begin{array}{l}4.60 \\
3.67 \\
4.48\end{array}$ \\
\hline$P L(1)$ & $\begin{array}{l}37 \\
38 \\
39\end{array}$ & $\begin{array}{l}34 \\
31 \\
31\end{array}$ & $\begin{array}{l}34 \\
33 \\
33\end{array}$ & $\begin{array}{l}33 \\
31 \\
36\end{array}$ & $\begin{array}{l}34 \\
30 \\
31\end{array}$ \\
\hline
\end{tabular}

observations indicate that accurate RSS-to-distance mapping requires the investigation of antenna propagation model as well as receiver's sensitivity at different directions.

\section{RELATED WORK}

The performance of ADV_CHs in large-scale deployments have been studied in [20] and [21]. Unfortunately, these studies did not consider the effect of factors such as noise and path loss. The authors in [22] showed that BLE-based microlocation can be used in an indoor environment with $0.27 \mathrm{~m}$ accuracy using LNSM. However, the authors conducted the experiments in a single indoor environment and without considering the effect of multipath. Another study [23] analyzed the RSS variation of ADV_CHs individually in one environment where the distance between the nodes is fixed. The authors in [24] have used LNSM for object tracking in an environment 25 meters long. The authors in [25] have used mobile phones to estimate path loss in one environment. They also presented a comparison of path loss for BLE and 802.11 propagation. The main shortcoming of these works is the lack of studying the effect of interference, environmental characteristics and multipath. A recent study [26] on modeling BLE's ADV_CHs for indoor positioning carried out a series of experiments in a library using iBeacon. Although the authors have used LNSM, they did not report the parameters of this model. This overview highlights the need for a comprehensive empirical evaluation of BLE's ADV_CHs in various environments.

\section{CONCLUSION}

In this paper, we presented an extensive evaluation and characterization of ADV_CHs in BLE standard. We first studied the mean and variations of noise floor in four different environments, and in particular, we showed the effect of 802.11 interference on ADV_CHs. The findings of this research provide additional evidence for the significance of individual channel characterization due to the various levels of interference on each channel. Next, the signal propagation characteristics of these channels have been investigated, and we extracted the parameters of LNSM through empirical measurements. The proposed models can be directly used in simulation tools as well as BLE-based applications such as IPS. Despite the merits and popularity of LNSM, we have identified the scenarios where hardware and environmental factors reduce the reliability of this model.

\section{REFERENCES}

[1] A. Al-Fuqaha, M. Guizani, M. Mohammadi, M. Aledhari, and M. Ayyash, "Internet of things: A survey on enabling technologies, protocols, and applications," IEEE Communications Surveys \& Tutorials, vol. 17, no. 4, pp. 2347-2376, 2015.

[2] N. Newman, "Apple ibeacon technology briefing," Journal of Direct, Data and Digital Marketing Practice, vol. 15, no. 3, pp. 222-225, 2014.

[3] "The Rise of Beacon Technology." [Online]. Available: http://blog. bluetooth.com/the-rise-of-beacon-technology

[4] “StickNFind." [Online]. Available: https://www.indiegogo.com/projects/ sticknfind-bluetooth-powered-ultra-small-location-stickers

[5] "TrackR." [Online]. Available: https://secure.thetrackr.com/

[6] "Estimote." [Online]. Available: https://estimote.com

[7] "Indoor Location Market by Component Deployment Mode, Application, Vertical, and Region - Global Forecast to 2022." [Online]. Available: https://www.marketsandmarkets.com/ Market-Reports/indoor-positioning-navigation-ipin-market-989.html

[8] S. Zurbes, W. Stahl, K. Matheus, and J. Haartsen, "Radio network performance of bluetooth," in Proceeding of the International Conference on communications (ICC), vol. 3. IEEE, 2000, pp. 1563-1567.

[9] H.-H. Liu and Y.-N. Yang, "Wifi-based indoor positioning for multifloor environment," in Proceedings of the IEEE Region 10 Conference (TENCON). IEEE, 2011, pp. 597-601.

[10] H. Zou, B. Huang, X. Lu, H. Jiang, and L. Xie, "A robust indoor positioning system based on the procrustes analysis and weighted extreme learning machine," IEEE Transactions on Wireless Communications, vol. 15, no. 2, pp. 1252-1266, 2016.

[11] C. Yang and H.-R. Shao, "Wifi-based indoor positioning," IEEE Communications Magazine, vol. 53, no. 3, pp. 150-157, 2015.

[12] R. Akl, D. Tummala, and X. Li, "Indoor propagation modeling at 2.4 ghz for ieee 802.11 networks." in Wireless and Optical Communications, 2006.

[13] B. Dezfouli, M. Radi, S. A. Razak, T. Hwee-Pink, and K. A. Bakar, "Modeling low-power wireless communications," Journal of Network and Computer Applications, vol. 51, pp. 102-126, 2015.

[14] B. Dezfouli, M. Radi, K. Whitehouse, S. A. Razak, and H.-P. Tan, "Cama: Efficient modeling of the capture effect for low-power wireless networks," ACM Transactions on Sensor Networks (TOSN), vol. 11, no. 1, p. 20, 2014.

[15] S. Chieochan, E. Hossain, and J. Diamond, "Channel assignment schemes for infrastructure-based 802.11 wlans: A survey," IEEE Communications Surveys \& Tutorials, vol. 12, no. 1, 2010.

[16] T. S. Rappaport et al., Wireless communications: principles and practice. prentice hall PTR New Jersey, 1996, vol. 2.

[17] A. R. Conn, N. I. Gould, and P. L. Toint, Trust region methods. Siam, 2000 , vol. 1.

[18] “nRF52840 Objective Prodcut spesification v0.5.1." [Online]. Available: http://infocenter.nordicsemi.com/pdf/nRF52840_OPS_v0.5.1.pdf

[19] A. Zanella and A. Bardella, "Rss-based ranging by multichannel rss averaging," IEEE Wireless Communications Letters, vol. 3, no. 1, pp. $10-13,2014$.

[20] Á. Hernández-Solana, D. Perez-Diaz-de Cerio, A. Valdovinos, and J. L. Valenzuela, "Proposal and evaluation of ble discovery process based on new features of bluetooth 5.0," Sensors, vol. 17, no. 9, p. 1988, 2017.

[21] A. F. Harris III, V. Khanna, G. Tuncay, R. Want, and R. Kravets, "Bluetooth low energy in dense iot environments," IEEE Communications Magazine, vol. 54, no. 12, pp. 30-36, 2016.

[22] F. Zafari and I. Papapanagiotou, "Enhancing ibeacon based microlocation with particle filtering," in Proceedings of the the Global Communications Conference (GLOBECOM). IEEE, 2015, pp. 1-7.

[23] R. Faragher and R. Harle, "Location fingerprinting with bluetooth low energy beacons," IEEE journal on Selected Areas in Communications, vol. 33, no. 11, pp. 2418-2428, 2015.

[24] G. Ionescu, C. M. de la Osa, and M. Deriaz, "Improving distance estimation in object localization with bluetooth low energy," SENSORCOMM, vol. 2014, pp. 45-50, 2014.

[25] M. Ji, J. Kim, J. Jeon, and Y. Cho, "Analysis of positioning accuracy corresponding to the number of ble beacons in indoor positioning system," in Processing of the Advanced Communication Technology (ICACT). IEEE, 2015, pp. 92-95.

[26] X. Wu, R. Shen, L. Fu, X. Tian, P. Liu, and X. Wang, "ibill: Using ibeacon and inertial sensors for accurate indoor localization in large open areas," IEEE Access, vol. 5, pp. 14589-14 599, 2017. 